Project No. 09-824

\title{
Utilization of Methacrylates and Polymer Matrices for the Synthesis of Ion Specific Resins
}

\section{Integrated University Programs}

Dr. Ken Czerwinski University of Nevada, Las Vegas

James Bresee, Federal POC George Goff, Technical POC

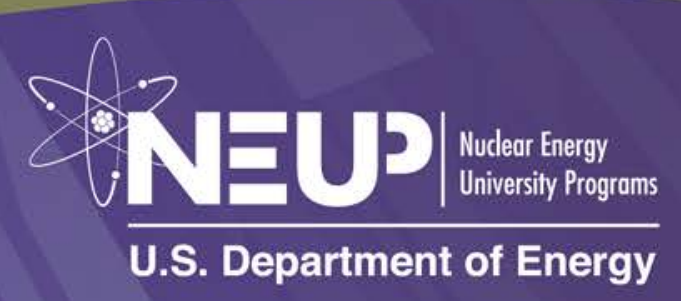


Utilization of Methacrylates and Polymer Matrices for the Synthesis of Ion Specific Resins (09-285): Quarterly and Final Report (July 2012 to September 2012)

Daniel B. Rego, Sierra Mayorga, Phil Weck, and Ken Czerwinski, University of Nevada, Las Vegas

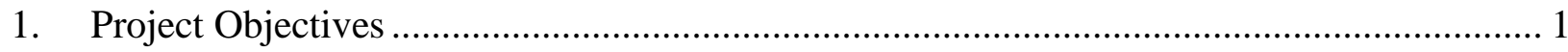

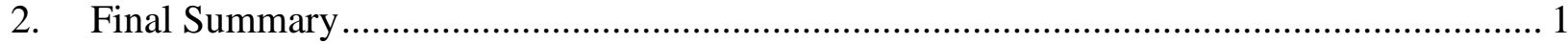

3. Path to Accomplish Scope …............................................................................... 2

3.1. Task 1: Synthesis of chain complexes ..................................................................... 2

3.2. Task 2: Evaluation of specific ligands and polymerization ........................................ 2

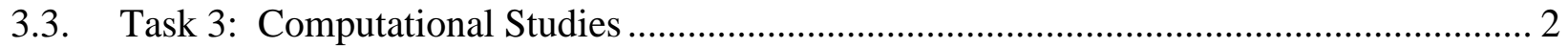

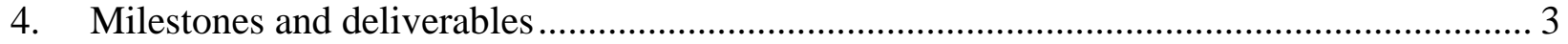

5. $\quad$ Work Performed Previous Quarter (April 2012 to June 2012)............................................ 3

6. Work Performed in Current Quarter (July 2012 to Sept 2012) ........................................... 4

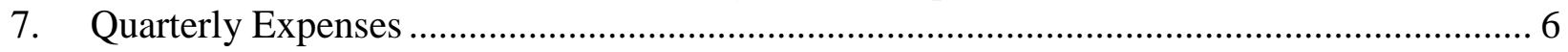

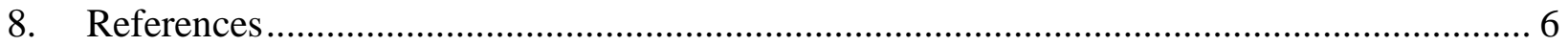

\section{Project Objectives}

Within advanced fuel cycle separations there are currently proposals for the disposal, storage, and/or transmutation of actinides such as Am, which will require the development of specific separation schemes. Existing efforts focus on solvent extraction systems for achieving suitable separation of actinide from lanthanides. However, previous work has shown the feasibility of ion-imprinting polymer-based resins for use in ion exchange type separations with metal ion recognition ${ }^{1}$. It has been demonstrated that phenolic-based resins function well for Am-Eu separations, but exhibited slow kinetics and difficulties in the imprinting process.

This proposal addresses the need for new and innovative methods for the selective separation of actinides through novel ion imprinted resins. The incorporation of metals into extended frameworks, including the possibility of 3-D polymerized matrices that can serve as a solid state template for specific resin preparation, will be explored. For example, an anhydrous trivalent $f$ element chain can be formed directly from a metal carbonate and methacrylic acid from water. ${ }^{2}$

From these simple coordination complexes, ${ }^{2,3,4}$ molecules of discrete size ${ }^{5,6,7}$ or shape ${ }^{8}$ can be formed via the utilization of coordinating ligands or by use of an anionic multi-ligand system incorporating methacrylate. Additionally, alkyl methyl methacrylates have been used successfully to create template nanospaces, which underscore their potential utility towards their use as 3-D polymerized matrices. ${ }^{9}$ This provides a unique route for the preparation of a specific metal ion template for the basis of ion-exchange separations. Such separations may prove to be excellent discriminators of metal ions, even between $f$-elements, as can be seen by the comparison of $\left\{\mathrm{Gd}\left[\mathrm{O}_{2} \mathrm{CC}\left(\mathrm{CH}_{2}\right) \mathrm{Me}\right]_{3}\right\}_{\mathrm{n}}{ }^{2}$ and $\left\{\mathrm{Eu}\left[\mathrm{O}_{2} \mathrm{CC}\left(\mathrm{CH}_{2}\right) \mathrm{Me}\right]_{3}\right\}_{\mathrm{n}}{ }^{3}$; even though the difference of the ionic radius between eight coordinate europium and gadolinium is only $0.011 \AA$, ${ }^{10}$ the smaller gadolinium shows contracted distances over $0.040 \AA$ smaller. ${ }^{2,3}$

\section{Final Summary}

Resins were prepared and evaluated for sorption behavior, column properties, and proton exchange capacity. The use of lanthanides to template the resins resulting the identification of a 
coordination change around Pm. This information can be used to tune the cavity size of the resin material to preferentially bind specific lanthanides or trivalent actinides.

The resin synthesis was achieved with the formation of lanthanide methacrylate infinite chains for all non-radioactive lanthanides directly from water This included a new anhydrous species with a variable 7/8-coordination environment. Lanthanide tris-methacrylates form as 9coordinate dihydrate infinite chains for the larger lanthanides, La from $\mathrm{Nd}$. In contrast anhydrous infinite chains are obtained for the smaller lanthanides as 8-coordinate for Sm to Dy. A mixed 8-coordinate/7-coordinate complex was found for Ho and Er, and 7-coordinate for Tm to Lu. The synthesis improved upon previous reports and demonstrated for all of the middle lanthanides. The synthesis of anhydrous infinite chain methacrylate structures for the smaller lanthanides has shown a new 7-coordinate species that indicates decreased crystallographic symmetry in the solid state.

\section{Path to Accomplish Scope}

\subsection{Task 1: Synthesis of chain complexes}

Synthesis of homometallic mononuclear, oligonuclear, and infinite chain complexes will be performed. The focus will be on the early lanthanide metal and will include preparation in the presences and absence of ligands. Proposed ligands include phenazine, phenanthroline, bipyridine, amines, isonitriles, among others. The preparation of heterometallic multinuclear and infinite chain complexes with and without the aforementioned ligands will also be pursued.

\subsection{Task 2: Evaluation of specific ligands and polymerization}

In this task different ligands will be examined. They include cyclopentadienes, substituted amides, aryl oxides, and scorpionate ligands. Evaluating the potential to polymerize or potentially polymerize the methycarylate both in situ and ex situ to form a more rigid architecture will also be performed. The selectivity of the prepared resins will be examined. The metal ions to be examined include early lanthanides, Am, and $\mathrm{Cm}$.

\subsection{Task 3: Computational Studies}

The dynamics of reactions involved in the metharylate complex formation and the separation processes will be studied -along with the thermal stability of the complexes- using massively-parallel $a b$ initio molecular dynamics (AIMD) simulations at finite temperature AIMD simulations will be performed using the Car-Parrinello method [4] with periodic boundary conditions applied in order to model the bulk behavior with a limited number of molecules. The reaction enthalpy and Gibbs free energy, $G$, will be calculated for different oxidation states of the complexes investigated and will help in deciding whether the complex formation will be favorable or not. The theoretical variation of Gibbs free energy for oxidation/reduction reactions will be compared directly with the values obtained from experiments. Complementary transition state calculations using the linear synchronous transit (LST) and quadratic synchronous transit (QST) methods will be carried out.

The proposed DFT calculations will investigate: 1) the structures and properties of the homometallic mononuclear, oligonuclear, and infinite chain complexes, as well as the reaction mechanisms governing their synthesis; 2) the role of specific ligands; 3) the possible polymerization of methacrylate species. Calculations will also simulate the imprinting process with Am(III), which is difficult to achieve experimentally, and test the potential of Sm(III), 
$\operatorname{Pr}(\mathrm{III})$, and $\mathrm{Nd}(\mathrm{III})$ for Am templating. Our computational findings and results will be fed back into the experimental effort in order to optimize synthetic routes, characterize the new methacrylate-bearing structures formed, and target more effective separations strategies and processes.

\section{Milestones and deliverables}

The merits and utility of using molecular architectures of methacrylate containing complexes will be evaluated and complexes containing discrete combinations of relevant lanthanides and actinides will be examined. The milestone we expect to achieve in the first year include the synthesis and characterization of various metal methacrylate complexes, which will add to our knowledge of lanthanide carboxylate chemistry, an area for which discoveries and incites are still being made. ${ }^{11,12}$

Additionally, the ability of heterometallic combinations and the synthetic requirements will not only add to the basic understanding of mixed-metal chemistry, but also provide insight and possible selective complexation of relevant metals for separations and processing.

Another major milestone we plan to achieve is in investigating polymerization via the methacrylate ligand, which could provide a way of investigating their possible use in exchange resins and columns. The utility of templated architecture in preparation for separations will be evaluation via this system. The ability of the prepared resin to selectively separation trivalent actinides will be assessed for comparison. These would not only add to the knowledge of separations via polymerized resins, but in their very synthesis, as well as the potential for developing new synthesis that incorporate the templating metal ion in a concomitant reaction.

For each year, an annual report to disseminate the achieved milestones shall be included, as well as quarterly reports to convey the rapidness of said achievements.

\section{Work Performed Previous Quarter (April 2012 to June 2012)}

Efforts in this quarter focused on the interaction of the resins, made from $\mathrm{Ln}\left[\mathrm{O}_{2} \mathrm{CC}\left(\mathrm{CH}_{2}\right) \mathrm{Me}\right]_{3}$ (aq.) $(\mathrm{Ln}=\mathrm{Nd}, \mathrm{Sm})$ without the use of copolymer, with samarium and neodymium solutions. These were examined by monitoring the interaction of the resins with lanthanide solutions using Near IR. The Near IR absorption bands of most of the lanthanides, including neodymium and samarium, are well suited to analytical methods due to a variety of strong absorption bands that are often distinct from each other, ${ }^{13}$ and neither neodymium nor samarium have overlapping or competing peaks in the range of 700-1400 nm. Neodymium, for example, was monitored using its strong bands at 742.5 and $868 \mathrm{~nm}(\varepsilon=6.22$ and 3.10, respectively), while samarium was monitored using its bands at 1095 and $1250 \mathrm{~nm}(\varepsilon=2.00$ and 2.19 , respectively). The resins tested were made as previously reported by UV irradiation (ca $300 \mathrm{~nm})$ of solutions of $\mathrm{Ln}\left[\mathrm{O}_{2} \mathrm{CC}\left(\mathrm{CH}_{2}\right) \mathrm{Me}_{3}(a q).(\mathrm{Ln}=\mathrm{Nd}\right.$, Sm) under slow r.t. evaporation of the water.

The resins were protonated using aqueous citric acid solutions $(2.650 \mathrm{~g}$ and $3.240 \mathrm{~g}$ of the monohydrate for the $1.698 \mathrm{~g}$ and $2.208 \mathrm{~g}$ of the samarium and neodymium resins respectively), and thereafter rinsed with deionized water. The resins were then converted to a sodium salt by addition of sodium hydroxide solutions $494 \mathrm{mg}$ and $601 \mathrm{mg}$ for the above samarium and neodymium templated resins, respectively. The material was allowed to dry under dynamic vacuum overnight.

A $0.16 \mathrm{M}$ solution of $\mathrm{Sm}\left(\mathrm{NO}_{3}\right)_{3}$ or $0.08 \mathrm{M}$ solution of $\mathrm{Nd}\left(\mathrm{NO}_{3}\right)_{3}$, 3mL aliquots each, showed no appreciable decrease in absorbance in the presence of the natriated sodium salt resins 
templated around neodymium, with $19.6 \mathrm{mg}$ and $17.6 \mathrm{mg}$ repectivly. Additionally, the A 3mL aliquot of $0.08 \mathrm{M}$ solution of $\mathrm{Sm}\left(\mathrm{NO}_{3}\right)_{3}$ did not show any decrease in absorption with the natriated sodium salt resin templated around samarium $(7.9 \mathrm{mg})$ was added. However, a $0.08 \mathrm{M}$ solution of $\mathrm{Nd}\left(\mathrm{NO}_{3}\right)_{3}$ (aq.) did show a decrease in absorbance at 742.5 and $868 \mathrm{~nm}$ of $c a 12 \%$ in the presense of $21.8 \mathrm{mg}$ of the natriated sodium salt resin templated around samarium. The $12 \%$ decrease is equivalent to $29 \mu \mathrm{mol}$. The resin had ca $67 \mu \mathrm{mol}$ equivalent maximum capacity (three $\mathrm{Na}^{+}$for each $\mathrm{Nd}^{3+}$ ) for pure sodium polymethacrylate. This would give ca $43 \%$ uptake of the theoretical maximum.

Additionally, studies of the resins by Scanning Electron Microscope (SEM) and Energy Dispersive Spectroscopy (EDS) utilizing a Joel JSM-5600 Scanning Electron Microscope continued. To improve reliability of the semi-quantitative EDS, and reduce problems with conductivity and the accumulation of electron charge, carbon coatings were applied to the resins made from samarium and neodymium without the presence of co-polymers. The EDS data was collected with INCA mapping software, and showed for both samples, a dispersion of the metals (samarium or neodymium) throughout the samples. This shows that the metal binding sites are not localized, nor are there appreciable area of polymerized resin that formed without the lanthanide templating metal.

The surface of the samarium templated material showed plate-like clusters. This stands in contrast with the needle-like formations of the infinite chains of $\left\{\operatorname{Sm}\left[\mu-\eta^{1}: \eta^{1-O}-\right.\right.$ $\left.\left.{ }_{2} \mathrm{CC}\left(\mathrm{CH}_{2}\right) \mathrm{Me}\right]\left[\mu-\eta^{2}: \eta^{1}-\mathrm{O}_{2} \mathrm{CC}\left(\mathrm{CH}_{2}\right) \mathrm{Me}\right]_{2}\right\}_{\mathrm{n}}$. The neodymium templated material did not show the distinct formation, plate-like or otherwise, though at greater magnification, the material was shown to not be homogenious.

The EDS data showed a wide disbursement of the samarium on average ca $45 \%$ by weight, which is considerably more than the $14 \%$ by weight seen in the resin when carbon coating is not used. The EDS data for the neodymium resin showed on average ca $50 \%$ by weight.

\section{Work Performed in Current Quarter (July 2012 to Sept 2012)}

Efforts in this quarter focused on optimizing the syntheses, and testing, of the resin material made from solutions of $\mathrm{Ln}\left[\mathrm{O}_{2} \mathrm{CC}\left(\mathrm{CH}_{2}\right) \mathrm{Me}\right]_{3}(a q).(\mathrm{Ln}=\mathrm{Nd}, \mathrm{Sm})$ irradiated by UV light (ca 300 $\mathrm{nm}$ ) under slow r.t. evaporation of the water. Sample without copolymer were made along with samples that utilized the co-polymers $\mathrm{Si}\left(\mathrm{CHCH}_{2}\right)_{4}$ and $\mathrm{O}\left[\mathrm{SiMe}_{2}\left(\mathrm{CHCH}_{2}\right)\right]_{2}$.

The quality of the irradiated material was found to be heavily dependent on the intensity of UV irradiation, the length of exposure, rate of evaporation, \&c., with more intense UV light and slower rates of evaporation (and thus length of exposure time) were found to provide the most robust material.

The resins were washed with $\mathrm{HCl}$ (aq.) in a 3:1 ratio with the lanthanide metal, and thereafter with deionized water, with the material co-polymerized with $\mathrm{O}\left[\mathrm{SiMe}_{2}\left(\mathrm{CHCH}_{2}\right)\right]_{2}$ most consistently giving the best yields ( $c$ 59-83\%). The material, after drying, was an off-white spongy material, Figure 1. Natriation with sodium was achieved by washing the protonated material with equimolar amounts of $\mathrm{NaOH}(a q$.$) . The resin material is not resilient with this$ method of sodium addition, with yields less than $50 \%$. 


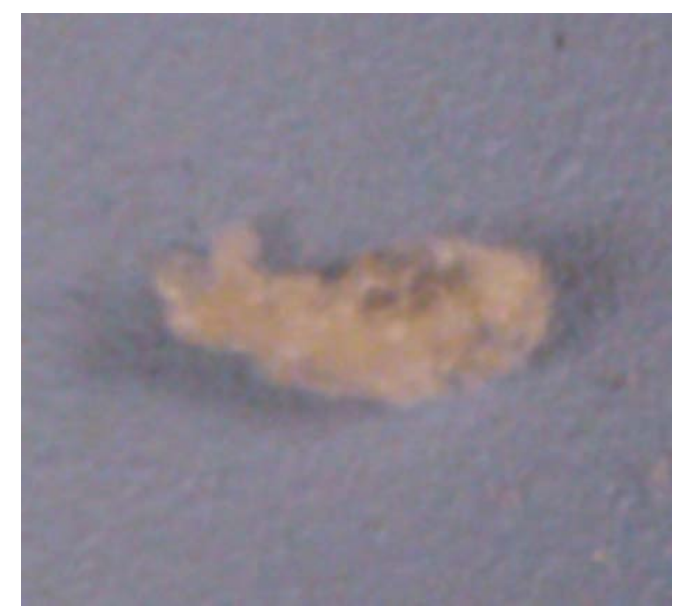

Figure 1. protonated resin template.

To examine the interaction of the resins with lanthanide solutions, the use of Near IR was chosen as a facile method for bulk small scale reactions. The Near IR absorption bands of most of the lanthanides, including neodymium and samarium, are well suited to analytical methods due to a variety of strong absorption bands which are often distinct from each other. ${ }^{14}$ Neodymium, for example, has strong bands at 742.5 and $868 \mathrm{~nm}(\varepsilon=6.22$ and 3.10, respectively), while samarium has bands at 1095 and $1250 \mathrm{~nm}(\varepsilon=2.00$ and 2.19, respectively); neither neodymium nor samarium have overlapping or competing peaks in the range of 700-1400 $\mathrm{nm}$. Furthermore, the absorption bands in the Near IR tend to be distinctive of the element itself rather than coordination environment dependent.

Resins were measured in three $\mathrm{pH}$ levels: 3, 4, 5, and 6. Each resin was presoaked in a $\mathrm{HNO}_{3}$ solution at the appropriate $\mathrm{pH}$. Mixed lanthanide solutions of praseodymium, neodymium, and samarium $(45 \mu \mathrm{M})$ had their $\mathrm{pH}$ adjusted by the addition of $\mathrm{HNO}_{3}$ or $\mathrm{NaOH}$ and monitored by a $\mathrm{pH}$. The resins were allowed to react over a period of 24 hours.

The samarium templated resins tended to uptake neodymium, while giving up samarium retained in the resins; the opposite was true for neodymium template resins. These results were most consistent at $\mathrm{pH} 5$ and 6, while at $\mathrm{pH} 4$ neodymium and samarium template resins would non-uniformly uptake their neodymium and samarium, respectively. For $\mathrm{pH} \mathrm{4,} \mathrm{5,} \mathrm{and} \mathrm{6,} \mathrm{all} \mathrm{resins}$ uptook, net, lanthanides from solution. The maximum percent uptake, relative to previously measured proton exchange capacities were $5.7 \%$ for the samarium templated resin with $\mathrm{O}\left[\mathrm{SiMe}_{2}\left(\mathrm{CHCH}_{2}\right)\right]_{2}$, and $6.7 \%$ for the neodymium templated resin with $\mathrm{Si}\left(\mathrm{CHCH}_{2}\right)_{4}$, Table 1 . In contrast, $\mathrm{pH} 3$ samples had little to no net uptake of lanthanides, and it is possible that higher $\mathrm{pH}$ levels could be degrading the resin material.

\begin{tabular}{|l|l|l|l|l|l|l|}
\hline $\mathrm{pH}$ & $\begin{array}{l}\text { Resin } \\
\text { templated } \\
\text { with Sm }\end{array}$ & $\begin{array}{l}\text { Resin } \\
\text { templated } \\
\text { with Sm \& } \\
\mathrm{Si}(\mathrm{CHCH})_{4}\end{array}$ & $\begin{array}{l}\text { Resin templated } \\
\text { with Sm \& } \\
\mathrm{O}\left[\mathrm{SiMe}_{2}\left(\mathrm{CHCH}_{2}\right)\right]_{2}\end{array}$ & $\begin{array}{l}\text { Resin } \\
\text { templated } \\
\text { with Nd \& } \\
\mathrm{Si}\left(\mathrm{CHCH}_{2}\right)_{4}\end{array}$ & $\begin{array}{l}\text { Resin templated } \\
\text { with Nd \& } \\
\mathrm{O}\left[\mathrm{SiMe}_{2}\left(\mathrm{CHCH}_{2}\right)\right]_{24}\end{array}$ & $\begin{array}{l}\text { Resin } \\
\text { templated } \\
\text { with Nd }\end{array}$ \\
\hline 3 & $0.53 \%$ & $-0.80 \%$ & $2.45 \%$ & $0.76 \%$ & $0.82 \%$ & $-0.11 \%$ \\
\hline 4 & $1.69 \%$ & $3.24 \%$ & $4.46 \%$ & $4.37 \%$ & $2.25 \%$ & $2.00 \%$ \\
\hline 5 & $2.50 \%$ & $2.82 \%$ & $5.69 \%$ & $1.71 \%$ & $6.65 \%$ & $3.22 \%$ \\
\hline 6 & $1.48 \%$ & $0.36 \%$ & $3.44 \%$ & $0.57 \%$ & $4.92 \%$ & $0.98 \%$ \\
\hline
\end{tabular}

Table 1. Actual uptake of lanthanides as percentage of theoretical based on proton exchange capacity. 
Praseodymium, which was not present in the templating of any of the resins, was uniformally taken up by all resins, with neodymium template resins showing greater removal of praseodymium from solution. For the neodymium template resins, the maximum uptake was $23.45 \%$ (average maximum uptake of $15.5 \%$ ); for samarium template resins, the maximum uptake was $10.95 \%$ (average maximum uptake of $8.08 \%$ ).

Specific trends for each resin across the four $\mathrm{pH}$ levels, or for each resin withing a certain $\mathrm{pH}$ level, were not consistently seen, though general trends could be seen: Lower $\mathrm{pH}$ levels gave better uptake and consistency than $\mathrm{pH} 3$, with $\mathrm{pH} 5$ being optimal; and the uptake of praseodymium was greatest with neodymium templated resins for all $\mathrm{pH}$ levels. Though the polymerization was highly sensitive to the polymerization conditions, and the resins were not yet fully regularized, a general ability to uptake lanthanides was shown a general greater demonstrated affinity of praseodymium for neodymium templated resins was seen.

\section{Quarterly Expenses}

\begin{tabular}{|c|r|r|r|r|r|}
\hline \multirow{2}{*}{ Q3 - Y3 } & July 2012 & Aug 2012 & Sept 2012 & QTR TOTAL & CUM TOTAL \\
\cline { 2 - 6 } \\
$\mathbf{4}$ /1/2012 -
\end{tabular}

\section{References}

1 Nishide, H.; Deguchi, J.; Tsuchida, E. Chem. Lett. 1976, 169-174.

2 Rego, Daniel B. Lanthanide Reduction Chemistry of the $L_{3} Z_{3} / M$ and $L n Z_{2} Z^{\prime} / M$ Systems and Related Coordination Chemistry Disseration, University of California, Irvine, 2007.

3 Petrochenkova, N.V.; Bukvetskii, B. V.; Mirochnik, A. G.; Karasev, V. E. Rus. J. Coord. Chem. 2002, 28, 67-73.

4 Birylina, V. V. Zhurnal Obshchei Khimii 1981, 7, 1467-1470.

5 Lu, W.; Luo, X.; Wu, B.; Mao, J.; Jiang, X. Acta Cryst. C 1999, 1472-1475.

6 Katkova, M. A.; Leonov, M. R.; Severina, T. V. Radiokhimiya 1992, 34, 17-21.

7 Katkova, M. A.; Leonov, M. A.; Severina, T. V. Metalloorganisheskaya Khimiya, 1992, 5, 1211.

8 Lu, W.-M.; Wu, J.-B.; Dong, N.; Chun, W.-G. Acta Cryst. C 1995, 1568-1570.

9 Serizawa, T.; Akashi, M. Polymer Journal 2006, 38, 311-328.

10 Shannon, R. D. Acta Cryst. 1976, A32, 751.

11 Davies, N. W.; Frey, A. S. P.; Gardiner, M. G.; Wang, J. Chem. Comm. 2006, 4853.

12 Evans, W. J.; Rego, D. B.; Ziller, J. W.; Rheingold, A. L.; DiPasquale, A. G. Organometallics, 2007, 26, 4737-4747.

13 Stewart, D.C.; Kato, D. Anal.Chem. 1958, 30, 164.

14 Stewart, D.C.; Kato, D. Anal.Chem. 1958, 30, 164. 